\title{
Steric control over molecular structure and supramolecular association exerted by tin- and ligand-bound groups in diorganotin carboxylates
}

Dainis Dakternieks, * and Andrew Duthie

Centre for Chiral and Molecular Technologies, Deakin University, Geelong, Victoria

3217, Australia

Douglas R. Smyth, and Clynton P.D. Stapleton

Department of Chemistry, The University of Adelaide, Australia 5005

Edward R.T. Tiekink*

Department of Chemistry, The University of Adelaide, Australia 5005; Present

address: Department of Chemistry, National University of Singapore, Singapore

117543

*** Supplementary Material ***

Details of yield, melting point, elemental analysis and IR data for $\mathbf{1}-\mathbf{8}$.

Figure S(1) Molecular structure for 1

Figure $\mathrm{S}(2)$ Molecular aggregation for $\mathbf{1}$

Figure S(3) Molecular structure for $\mathbf{3}$

Figure S(4) Molecular structure for 4

Figure S(5) Molecular structure for 7

Figure S(6) Molecular structure for 8 
Details of yield, melting point, elemental analysis and IR data for $\mathbf{1}-\mathbf{8}$.

$\mathrm{Me}_{2} \mathrm{Sn}(2 \text {-pic) })_{2}$, (1): $91 \%$ yield; mp 264-266 ${ }^{\circ} \mathrm{C}$. Anal. Calcd for $\mathrm{C}_{14} \mathrm{H}_{14} \mathrm{~N}_{2} \mathrm{O}_{4} \mathrm{Sn}$ :

C, 42.79; H, 3.59; N, 7.13. Found: C, 42.45; H, 3.50; N, 7.20. IR: v(C=O) 1675 (s);

$v(\mathrm{C}-\mathrm{O}) 1348(\mathrm{~s}) ; \mathrm{v}(\mathrm{COO})_{\text {asymm }}$ 1625(s); $v(\mathrm{COO})_{\text {symm }} 1386(\mathrm{~m}) \mathrm{cm}^{-1}$.

$\mathbf{n B u}{ }_{2} \mathbf{S n}(2-p i c)$, (2): $78 \%$ yield; mp 192-195 ${ }^{\circ} \mathrm{C}$. Anal. Calcd for

$\mathrm{C}_{20} \mathrm{H}_{26} \mathrm{~N}_{2} \mathrm{O}_{4} \mathrm{Sn}: \mathrm{C}, 50.35 ; \mathrm{H}, 5.49 ; \mathrm{N}, 5.87$. Found: C, 50.35; H, 5.35; N, 6.15. IR:

$v(\mathrm{C}=\mathrm{O}) 1669(\mathrm{~s}) ; \mathrm{v}(\mathrm{C}-\mathrm{O}) 1346(\mathrm{~s}) ; \mathrm{v}(\mathrm{COO})_{\mathrm{asymm}}$ 1627(s); v(COO) $)_{\text {symm }} 1383(\mathrm{~s}) \mathrm{cm}^{-1}$.

$\mathbf{t B u}_{\mathbf{2}} \mathbf{S n}\left(\mathbf{2}\right.$-pic) ${ }_{2}$, (3): 87\% yield; mp 210-212 ${ }^{\circ} \mathrm{C}$. IR: v(C=O) 1669 (vs), 1655

(vs); v(C-O) 1328 (vs) cm ${ }^{-1}$. Literature compound: Jurkschat, K.; Tiekink, E. R. T.

Main Group Metal Chem. 1994, 17, 659.

$\mathbf{P h}_{2} \mathbf{S n}(2-p i c)$, (4): $50 \%$ yield; $\mathrm{mp}>250{ }^{\circ} \mathrm{C}$ (dec.). Anal. Calcd for

$\mathrm{C}_{24} \mathrm{H}_{18} \mathrm{~N}_{2} \mathrm{O}_{4} \mathrm{Sn}: \mathrm{C}, 55.75 ; \mathrm{H}, 3.51 ; \mathrm{N}, 5.42$. Found: C, 55.55; H, 3.45; N, 5.50. IR:

$v(\mathrm{C}=\mathrm{O}) 1678(\mathrm{~s}) ; \mathrm{v}(\mathrm{C}-\mathrm{O}) 1331(\mathrm{~s}) \mathrm{cm}^{-1}$.

$\mathbf{C y}_{2} \mathbf{S n}(2-p i c)_{2}\left(\mathbf{O H}_{2}\right)$, (5): $94 \%$ yield; mp 226-228 ${ }^{\circ} \mathrm{C}$. Anal. Calcd for

$\mathrm{C}_{24} \mathrm{H}_{30} \mathrm{~N}_{2} \mathrm{O}_{4}$ Sn. $\mathrm{OH}_{2}$ : C, 52.67; H, 5.90; N, 5.12. Found: C, 53.00; H, 5.65; N, 5.15.

IR: v(C=O) $1680(\mathrm{~s}), 1654(\mathrm{~s}) ; v(\mathrm{C}-\mathrm{O}) 1344(\mathrm{~s}) ; \mathrm{v}(\mathrm{COO})_{\mathrm{asymm}} 1623(\mathrm{~s}) ; \mathrm{v}(\mathrm{COO})_{\mathrm{symm}}$ $1375(\mathrm{~s}) \mathrm{cm}^{-1}$.

$\operatorname{Me}_{2} \operatorname{Sn}\left(2\right.$-quin)2, (6): $93 \%$ yield; $m p$ 268-270 ${ }^{\circ} \mathrm{C}$. Anal. Calcd for

$\mathrm{C}_{22} \mathrm{H}_{18} \mathrm{~N}_{2} \mathrm{O}_{4} \mathrm{Sn}: \mathrm{C}, 53.59 ; \mathrm{H}, 3.68 ; \mathrm{N}$, 5.68. Found: C, 53.54; H, 3.58; N, 5.61. IR:

$v(\mathrm{C}=\mathrm{O}) 1677(\mathrm{~s}), 1665(\mathrm{~s}) ; \mathrm{v}(\mathrm{C}-\mathrm{O}) 1320(\mathrm{~s}) \mathrm{cm}^{-1}$.

$\mathrm{tBu}_{2} \mathbf{S n}\left(2\right.$-quin) ${ }_{2}$, (7): 55\% yield; mp 202-204 ${ }^{\circ} \mathrm{C}$. Anal. Calcd for

$\mathrm{C}_{28} \mathrm{H}_{30} \mathrm{~N}_{2} \mathrm{O}_{4} \mathrm{Sn}: \mathrm{C}, 58.26 ; \mathrm{H}, 5.24 ; \mathrm{N}, 4$.85. Found: $\mathrm{C}, 58.45 ; \mathrm{H}, 5.25 ; \mathrm{N}, 4.80$. IR:

$v(\mathrm{C}=\mathrm{O}) 1670$ (vs), 1655 (vs); v(C-O) 1326 (vs) $\mathrm{cm}^{-1}$. 
$\mathrm{Cy}_{2} \mathbf{S n}$ (2-quin)2, (8): $73 \%$ yield; mp $234236^{\circ} \mathrm{C}$. Anal. Calcd for $\mathrm{C}_{20} \mathrm{H}_{26} \mathrm{~N}_{2} \mathrm{O}_{4} \mathrm{Sn}: \mathrm{C}, 61.07 ; \mathrm{H}, 5.45 ; \mathrm{N}, 4$. 45. Found: $\mathrm{C}, 60.30 ; \mathrm{H}, 5.45 ; \mathrm{N}, 4.30$. IR: $v(\mathrm{C}=\mathrm{O}) 1668(\mathrm{vs}) ; \mathrm{v}(\mathrm{C}-\mathrm{O}) 1326(\mathrm{vs}) \mathrm{cm}^{-1}$. 


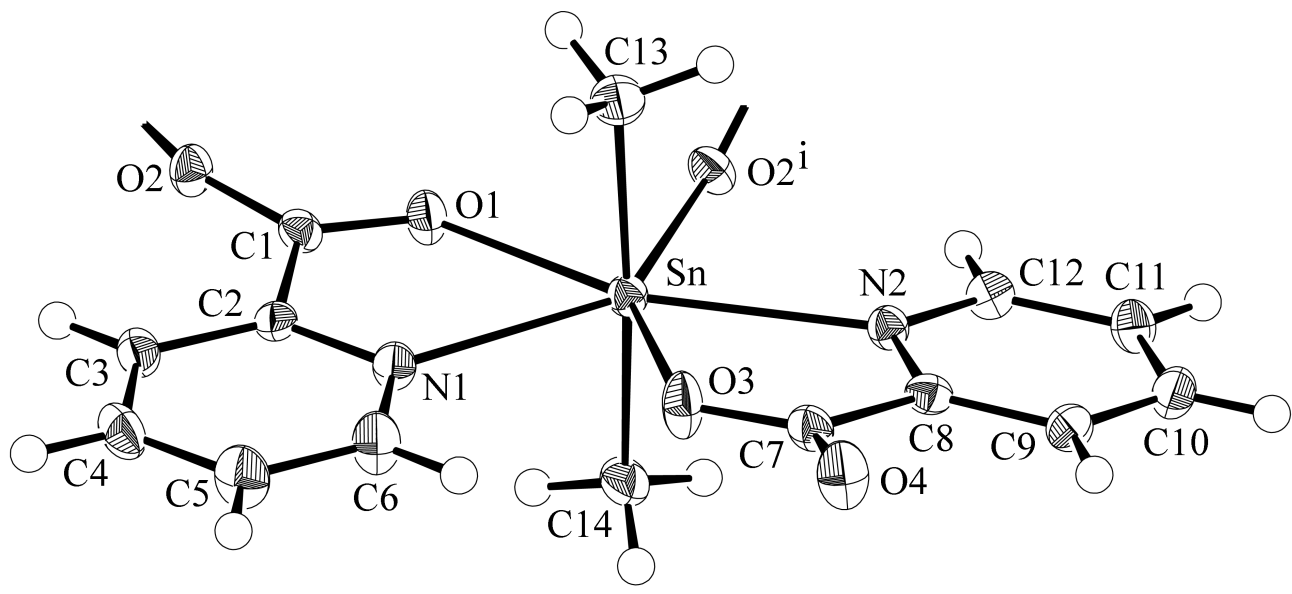

Figure S(1) Molecular structure for $\mathbf{1}$ 


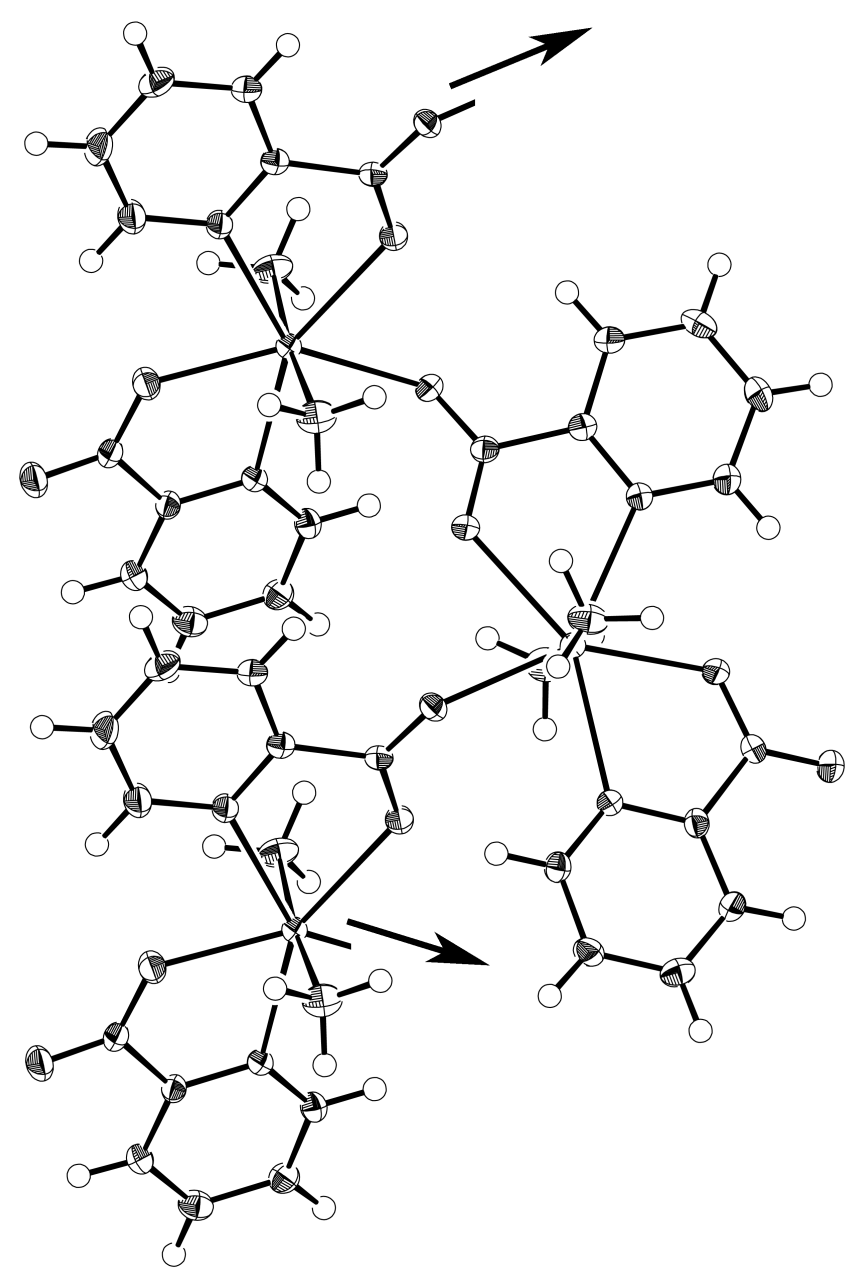

Figure S(2) Molecular aggregation for $\mathbf{1}$ 


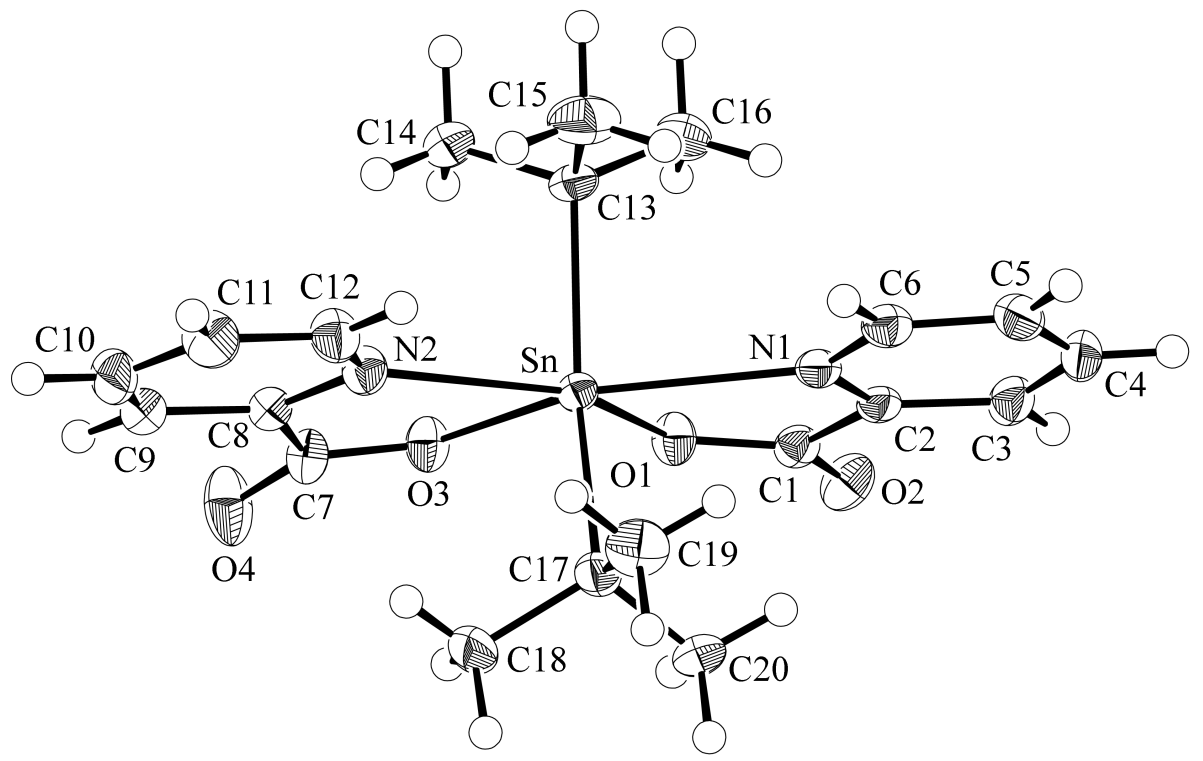

Figure S(3) Molecular structure for 3 


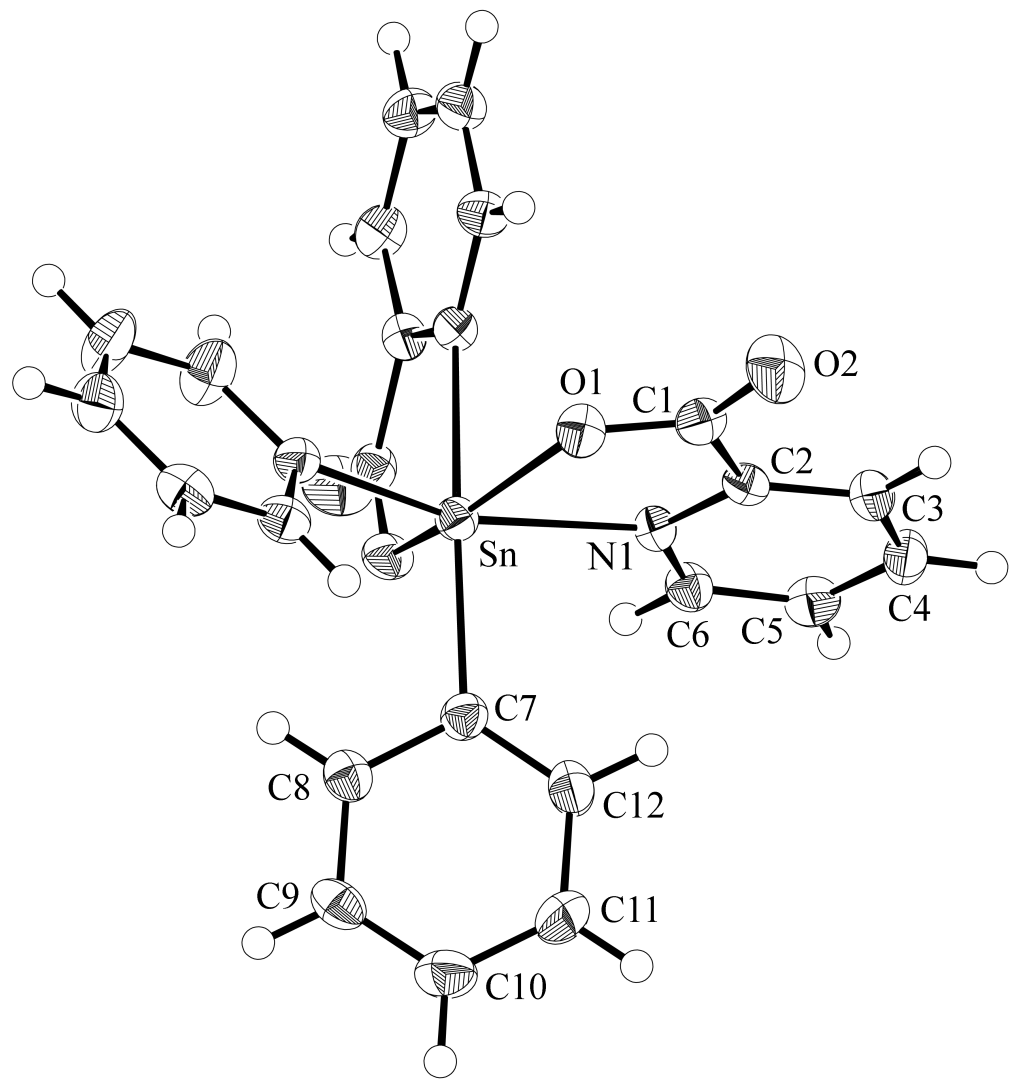

Figure S(4) Molecular structure for 4 


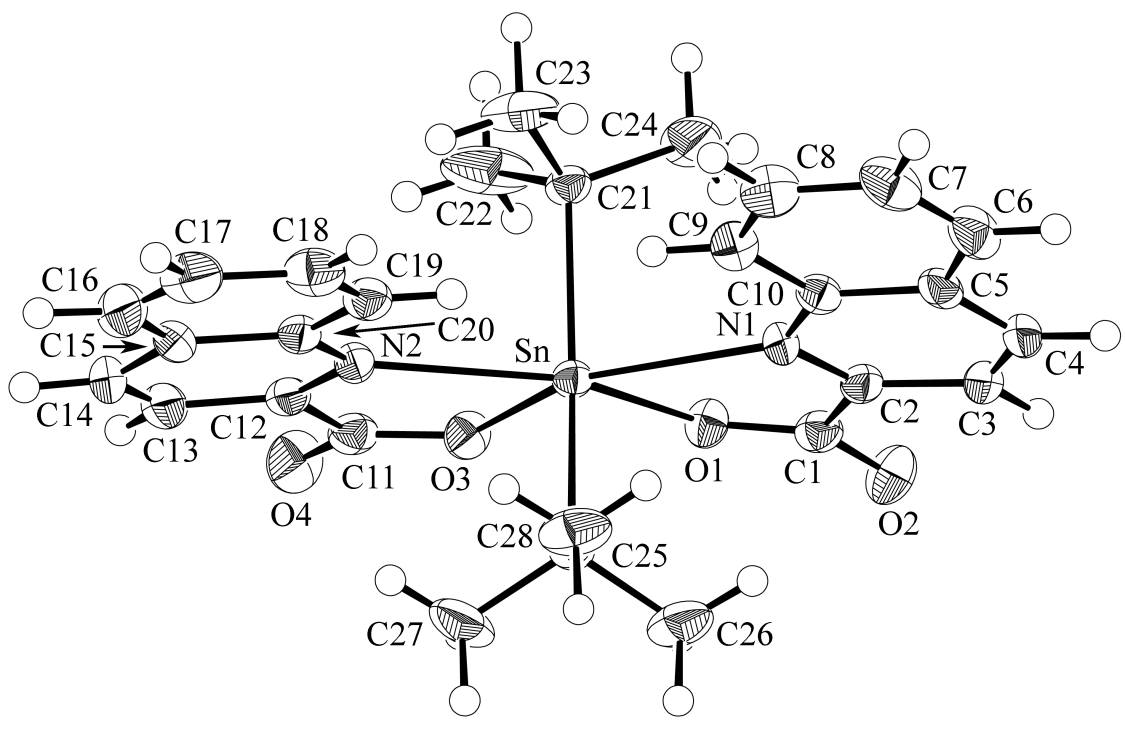

Figure S(5) Molecular structure for 7 


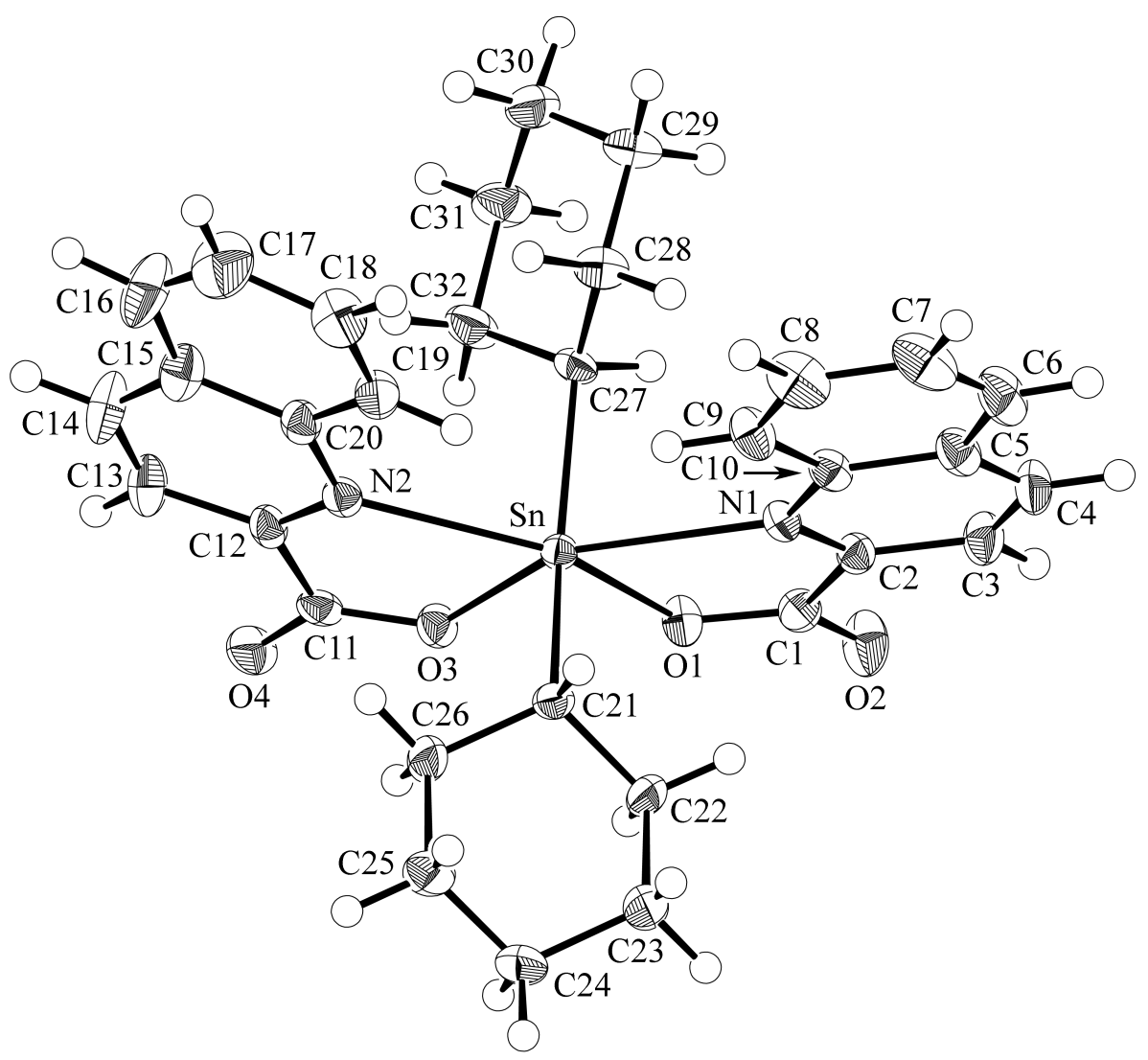

Figure S(6) Molecular structure for 8 